\title{
Addendum: Five-loop renormalisation of QCD in covariant gauges
}

\author{
K.G. Chetyrkin, ${ }^{a}$ G. Falcioni, ${ }^{b}$ F. Herzog ${ }^{b}$ and J.A.M. Vermaseren ${ }^{b}$ \\ ${ }^{a}$ II Institut für Theoretische Physik, Universität Hamburg, \\ Luruper Chaussee 149, 22761 Hamburg, Germany \\ ${ }^{b}$ Nikhef Theory Group, \\ Science Park 105, 1098 XG Amsterdam, The Netherlands \\ E-mail: konstantin.chetyrkin@desy.de, gfalcion@nikhef.nl, \\ fherzog@nikhef.nl, t68@nikhef.nl
}

AdDENDUM TO: JHEP10(2017)179

Keywords: Perturbative QCD, Renormalization Group

ARXIV EPRINT: 1709.08541

Due to an oversight the authors regret not including several references where parts of their five-loop results were presented. They confirm that the relevant leading order and next-toleading order coefficients of the polynomial in $n_{f}$ of the gluon, ghost and quark anomalous dimensions as well as the beta-function are in agreement with the results of [1-3]. The authors would further like to cite [4] which is important in the establishment of the relation eq. (4.10) and in the computation of the large $n_{f}$ contribution to the anomalous dimension of the operator $A^{2}$ presented in eq. (4.11).

Open Access. This article is distributed under the terms of the Creative Commons Attribution License (CC-BY 4.0), which permits any use, distribution and reproduction in any medium, provided the original author(s) and source are credited.

\section{References}

[1] J.A. Gracey, Quark, gluon and ghost anomalous dimensions at $O\left(1 / N_{f}\right)$ in quantum chromodynamics, (1993), [https://doi.org/10.1016/0370-2693(93)91803-U].

[2] J.A. Gracey, The QCD $\beta$-function at $O\left(1 / N_{f}\right)$, Phys. Lett. B 373 (1996) 178 [hep-ph/9602214] [INSPIRE].

[3] M. Ciuchini, S.E. Derkachov, J.A. Gracey and A.N. Manashov, Computation of quark mass anomalous dimension at $O\left(1 / N_{f}^{2}\right)$ in quantum chromodynamics, Nucl. Phys. B 579 (2000) 56 [hep-ph/9912221] [INSPIRE].

[4] D. Dudal, J.A. Gracey, V.E.R. Lemes, R.F. Sobreiro, S.P. Sorella and H. Verschelde, Renormalization properties of the mass operator $A_{\mu}^{a} A_{\mu}^{a}$ in three dimensional Yang-Mills theories in the Landau gauge, Annals Phys. 317 (2005) 203 [hep-th/0409254] [INSPIRE]. 